\title{
MINIMAL FIRST COUNTABLE TOPOLOGIES
}

\author{
BY \\ R. M. STEPHENSON, JR.
}

1. Introduction. If $\mathscr{P}$ is a property of topologies, a space $(X, \mathscr{T})$ is $\mathscr{P}$-minimal or minimal $\mathscr{P}$ if $\mathscr{T}$ has property $\mathscr{P}$, but no topology on $X$ which is strictly weaker (=smaller) than $\mathscr{T}$ has $\mathscr{P} .(X, \mathscr{T})$ is $\mathscr{P}$-closed if $\mathscr{T}$ has property $\mathscr{P}$ and $(X, \mathscr{T})$ is a closed subspace of every $\mathscr{P}$-space in which it can be embedded.

$\mathscr{P}$-minimal and $\mathscr{P}$-closed spaces have been investigated for the cases $\mathscr{P}=$ Hausdorff, regular, Urysohn, completely Hausdorff, completely regular, locally compact, zero-dimensional, normal, completely normal, paracompact, and metric. A well-known result is that for any of these properties, a compact $\mathscr{P}_{\text {-space is minimal }}$ $\mathscr{P}$. If $\mathscr{P}=$ Hausdorff [6], Urysohn [13], regular [13], or completely Hausdorff [14], $\mathscr{P}$-minimality is a sufficient, but not a necessary condition for $\mathscr{P}$-closedness. If $\mathscr{P}=$ completely regular [4], [6], normal [4], [6], [16], zero-dimensional [3], locally compact [4], [6], completely normal [14], paracompact [14], or metric [14], then on any $\mathscr{P}$-space $\mathscr{P}$-minimality, $\mathscr{P}$-closedness, and compactness are equivalent conditions.

In this paper, we consider first countable- and $\mathscr{P}$-minimal spaces and first countable- and $\mathscr{P}$-closed spaces. A space $(X, \mathscr{T})$ is called first countable- and $\mathscr{P}$ minimal if $\mathscr{T}$ is first countable and has property $\mathscr{P}$, and if no first countable topology on $X$ which is strictly weaker than $\mathscr{T}$ has property $\mathscr{P}(X, \mathscr{T})$ is first countable- and $\mathscr{P}$-closed if $\mathscr{T}$ is first countable and has property $\mathscr{P}$, and $(X, \mathscr{T})$ is a closed subspace of every first countable $\mathscr{P}$-space in which it can be embedded.

We obtain several characterizations of such spaces, and we prove that for most of the above properties, the following statements are equivalent for a $\mathscr{P}$-space $X$ : $X$ is first countable- and $\mathscr{P}$-minimal; $X$ is first countable- and $\mathscr{P}$-closed; $X$ is first countable- and Hausdorff-minimal. A proof is given that if $\mathscr{P}$ denotes either completely regular or zero-dimensional, then a first countable $\mathscr{P}$-space is first countable- and $\mathscr{P}$-minimal if and only if it is pseudocompact. It is shown that if $\mathscr{P}=$ weakly normal, normal, completely normal, or paracompact, then a first countable $\mathscr{P}$-space is first countable- and $\mathscr{P}$-minimal if and only if it is countably compact. A proof is also given that a perfectly normal space is minimal perfectly normal if and only if it is countably compact. We prove that if $\mathscr{P}=$ Hausdorff, regular, completely regular, zero-dimensional, or weakly normal, and if $\{X(n)\}$ is a sequence of topological spaces, then $\Pi X(n)$ is first countable- and $\mathscr{P}$-minimal if and only if each $X(n)$ is first countable- and $\mathscr{P}$-minimal. We investigate which subspaces

Presented to the Society, January 25, 1969; received by the editors December 22, 1967 and, in revised form, April 9, 1968. 
of first countable- and $\mathscr{P}$-minimal spaces are first countable- and $\mathscr{P}$-minimal, and we prove that every first countable (semiregular) Hausdorff space can be embedded (densely) in a first countable- and Hausdorff-minimal space.

We use the same terminology as that in [8] or [10]. Thus, as used here, every separation property includes Hausdorff separation.

$R(N)$ will denote the set of real (natural) numbers. We shall denote the set of continuous mappings of a space $X$ into a space $Y$ by $C(X, Y)$. If $\mathscr{B}$ is a collection of sets, the set of all finite intersections of elements of $\mathscr{B}$ will be denoted by $\mathscr{B} \wedge$. We shall say that $\mathscr{B}$ is fixed (free) provided that $\bigcap \mathscr{B} \neq \varnothing(\bigcap \mathscr{B}=\varnothing)$.

\section{Characterizations.}

DEFINITION 2.1. A filter base on a topological space is called open (closed) provided that the sets belonging to it are open (closed). A regular filter base is an open filter base which is equivalent with a closed filter base.

DEFINITION 2.2. A topological space $X$ is feebly compact if every countable open filter base on $X$ has an adherent point.

Definition 2.3. Given a space $(X, \mathscr{T}),\left\{\operatorname{Int}_{\mathscr{T}} \mathrm{Cl}_{\mathscr{T}} T \mid T \in \mathscr{T}\right\}$ is a base for a topology $\mathscr{T}_{s}$ on $X .(X, \mathscr{T})$ is called semiregular if $\mathscr{T}_{s}=\mathscr{T}$.

THEOREM 2.4. Let $X$ be a first countable Hausdorff space. The following are equivalent:

(i) $X$ is first countable- and Hausdorff-minimal.

(ii) $X$ is semiregular and feebly compact.

(iii) Every countable open filter base on $X$ which has a unique adherent point is convergent.

THEOREM 2.5. Let $(X, \mathscr{T})$ be a first countable Hausdorff space. The following are equivalent:

(i) $(X, \mathscr{T})$ is first countable- and Hausdorff-closed.

(ii) $(X, \mathscr{T})$ is feebly compact.

(iii) $\left(X, \mathscr{T}_{s}\right)$ is first countable- and Hausdorff-minimal.

The proofs of Theorems 2.4 and 2.5 are omitted, for they are entirely similar to the proofs of corresponding statements about minimal Hausdorff spaces and absolutely closed spaces (see [8, pp. 167-168]).

THEOREM 2.6. Let $X$ be a first countable regular space. The following are equivalent:

(i) $X$ is first countable- and regular-minimal.

(ii) Every countable regular filter base on $X$ which has a unique adherent point is convergent.

(iii) Every countable regular filter base on $X$ has an adherent point.

(iv) Every countable regular filter base on $X$ is fixed.

(v) $X$ is first countable- and regular-closed.

(vi) $X$ is feebly compact.

(vii) $X$ is first countable- and Hausdorff-minimal. 
Proofs that (i) and (ii) are equivalent, that (iii) and (v) are equivalent, and that (ii) implies (iii) are similar to proofs of results in [7] and [13] concerning minimal regular spaces and regular-closed spaces. (iii) and (iv) are equivalent, because for any regular filter base $\mathscr{B}, \bigcap \mathscr{B}=\bigcap\{\bar{B} \mid B \in \mathscr{B}\}$. In [17, Theorem 2.6] the author proved that a regular space is feebly compact if and only if (iv) holds. If (vi) holds, then $X$ satisfies (ii) of Theorem 2.4 and hence is first countable- and Hausdorffminimal. (vii) obviously implies (i).

DEFINITION 2.7. An open filter base $\mathscr{B}$ on a space $X$ is called completely regular if for each $B \in \mathscr{B}$ there exist a set $C \in \mathscr{B}$ and a function $f \in C(X,[0,1])$ such that $f(C)=0$ and $f(X-B)=1$.

LEMMA 2.8. Let $(X, \mathscr{T})$ be a completely regular space, and let $\mathscr{C}$ be a countable completely regular filter base on $(X, \mathscr{T})$ which is free. Fix a point $p \in X$ and let $\mathscr{T}^{\prime}$ be the topology on $X$ which has as a base $\mathscr{T} \mid(X-\{p\}) \cup\{T \cup C \mid p \in T, T \in \mathscr{T}$, and $C \in \mathscr{C}\}$. Then $\mathscr{T}^{\prime}$ is a completely regular topology on $X$ which is strictly weaker than $\mathscr{T}$, and $\left(X, \mathscr{T}^{\prime}\right)$ is first countable if and only if $(X, \mathscr{T})$ is first countable.

Proof. Obviously $\left(X, \mathscr{T}^{\prime}\right)$ is first countable if and only if $(X, \mathscr{T})$ is first countable. Since $\mathscr{C}$ is a free regular filter base on $(X, \mathscr{T}), \mathscr{C}$ has no adherent points in $(X, \mathscr{T})$. Thus $\left(X, \mathscr{T}^{\prime}\right)$ is Hausdorff. $\mathscr{T}^{\prime}$ is weaker than $\mathscr{T}$ since $\mathscr{C}$ is an open filter base on $(X, \mathscr{T})$. If $T$ is an open neighborhood of $p$ in $(X, \mathscr{T})$ which is disjoint from a set $C \in \mathscr{C}$, then $T$ contains no neighborhood of $p$ in $\left(X, \mathscr{T}^{\prime}\right)$. Therefore, $\mathscr{T}^{\prime}$ is strictly weaker than $\mathscr{T}$.

To see that $\left(X, \mathscr{T}^{\prime}\right)$ is completely regular, consider a point $x \in X$ and an open neighborhood $N$ of $x$ in $\left(X, \mathscr{T}^{\prime}\right)$.

Case 1. $x \neq p$. Since $x$ is not an adherent point of $\mathscr{C}$ in $(X, \mathscr{T})$, there is a neighborhood $M$ of $x$ in $X-\{p\}$ so that for some $C \in \mathscr{C}, M \cap C=\varnothing$. Let $O=M \cap N$. Since $(X, \mathscr{T})$ is completely regular, there is a function $f \in C((X, \mathscr{T}),[0,1])$ which vanishes at $x$ and equals 1 on $X-O$. Clearly, $f$ is continuous on $\left(X, \mathscr{T}^{\prime}\right), f(x)=0$, and $f(X-N)=1$.

Case 2. $x=p$. Choose $T \in \mathscr{T}$ and $C \in \mathscr{C}$ so that $x \in T$ and $N \supset T \cup C$. Then there exist continuous mappings $f, g$ of $(X, \mathscr{T})$ into $[0,1]$ and a set $D \in \mathscr{C}$ such that $f(x)=0, f(X-T)=1, g(D)=0$, and $g(X-C)=1$. Let $h=f \wedge g$. Then

$$
h \in C\left(\left(X, \mathscr{T}^{\prime}\right),[0,1]\right), h(x)=0,
$$

and $h(X-N)=1$.

THEOREM 2.9. Let $X$ be a first countable completely regular space. The following are equivalent:

(i) $X$ is first countable- and completely regular-minimal.

(ii) $X$ is first countable- and completely regular-closed.

(iii) $X$ is pseudocompact.

(iv) $X$ is feebly compact.

(v) Every countable completely regular filter base on $X$ is fixed. 
Proof. (i) implies (ii). Let $Y$ be a first countable completely regular space such that $X \subset Y$ and suppose that $q \in \bar{X}$. Let $\mathscr{D}$ be a countable fundamental system of open neighborhoods of $q$. Then $\mathscr{C}=\mathscr{D} \mid X$ is a countable completely regular filter base on $X$, so it follows from Lemma 2.8 that there is a point $x \in \bigcap \mathscr{C}$. Since $Y$ is Hausdorff, $x$ must be $q$. Thus $q \in X$ and $X=\bar{X}$.

It follows from Theorems 2.3 and 2.7 in [17] that (ii), (iii), and (v) are equivalent. It is well known that (iii) and (iv) are equivalent [11]. According to Theorem 2.6, (iv) implies that $X$ is first countable- and Hausdorff-minimal. Thus (iv) implies (i).

LEMMA 2.10. Let $X$ be a first countable completely regular space, and let $\mathscr{C}$ be $a$ countable completely regular filter base on $X$ which is free. Take $p \notin X$, let $Y=X$ $\cup\{p\}$, and call a set $O \subset Y$ open if and only if (a) $O \cap X$ is open in $X$, and (b) if $p \in O$, then $O$ contains a set belonging to $\mathscr{C}$. The following hold:

(i) $Y$ is a first countable completely regular space in which $X$ is embedded as a proper dense subset.

(ii) If $X$ is paracompact (weakly normal, normal, completely normal, perfectly normal), then so is $Y$.

Proof. (i) is an immediate consequence of the proof of Theorem 2.7 in [17].

(ii) If $X$ is paracompact, then it follows from Proposition 5.3 in [9] that $Y$ is paracompact.

Suppose that $X$ is weakly normal, and let $F$ and $C$ be two closed subsets of $Y$ such that $C$ is countable and $F \cap C=\varnothing$. If $p \notin F \cup C$, then there are disjoint sets $O$ and $P$ open in $X$ such that $F \subset O$ and $C \subset P$. Since $X$ is open in $Y, O$ and $P$ are also open in $Y$. Suppose that $p \in F \cup C$, say $p \in F$. Then $F-\{p\}$ and $C$ are disjoint closed subsets of $X$, one of which is countable, so there are disjoint sets $M$ and $N$ open in $X$ such that $F-\{p\} \subset M$ and $C \subset N$. Since $Y$ is regular, there are disjoint sets $A$ and $B$ open in $Y$ such that $p \in A$ and $C \subset B$. Set $O=A \cup M$ and $P=N \cap B$. Then $O$ and $P$ are open in $Y, F \subset O, C \subset P$, and $O \cap P=\varnothing$.

The other cases are similar.

LeMmA 2.11. Let $X, \mathscr{T}, \mathscr{C}, \mathscr{T}^{\prime}$, and p be as in Lemma 2.8. If $(X, \mathscr{T})$ is paracompact (weakly normal, normal, completely normal, perfectly normal), then so is $\left(X, \mathscr{T}^{\prime}\right)$.

Proof. In [14] it was proved that if $(X, \mathscr{T})$ is paracompact and $\mathscr{C}$ is a free, countable, regular filter base on $(X, \mathscr{T})$, then $\left(X, \mathscr{T}^{\prime}\right)$ is paracompact. The same proof can be used here.

Suppose that $(X, \mathscr{T})$ is weakly normal, and consider two closed subsets $A$ and $B$ of $\left(X, \mathscr{T}^{\prime}\right)$ such that $A$ is countable and $A \cap B=\varnothing$. Since $(X, \mathscr{T})$ is weakly normal, there are disjoint sets $O, P \in \mathscr{T}$ such that $A \subset O$ and $B \subset P$.

Case 1. $p \notin A \cup B$. Let $K=O-\{p\}$ and $L=P-\{p\}$. Then $K, L \in \mathscr{T}^{\prime}, K \cap L=\varnothing$, $A \subset K$, and $B \subset L$.

Case 2. $p \in A \cup B$, say $p \in A$. Since $p \notin B$ and $\left(X, \mathscr{T}^{\prime}\right)$ is regular, there are disjoint sets $M, N \in \mathscr{T}^{\prime}$ such that $p \in M$ and $B \subset N$. Let $K=M \cup O$ and $L=N \cap P$. Then $A \subset K, B \subset L, K, L \in \mathscr{T}^{\prime}$, and $K \cap L=\varnothing$. 
The other cases are similar.

Lemma 2.12. Let $(X, \mathscr{T})$ be a first countable Hausdorff space which is countably compact. Then $(X, \mathscr{T})$ is a first countable- and Hausdorff-minimal regular space.

Proof. Let $\mathscr{R}$ be a first countable Hausdorff topology on $X$ such that $\mathscr{R} \subset \mathscr{T}$, and let $i$ be the identity mapping of $(X, \mathscr{T})$ onto $(X, \mathscr{R})$. Since $i$ is continuous and oneto-one, it follows from Example 3 of $[10$, p. 253] that $i$ is a homeomorphism.

It is well known that every first countable, countably compact, Hausdorff space is regular.

THEOREM 2.13. Let $\mathscr{P}$ denote any one of paracompact, weakly normal, normal, and completely normal, and let $(X, \mathscr{T})$ be a first countable $\mathscr{P}$-space. The following are equivalent:

(i) $(X, \mathscr{T})$ is first countable- and $\mathscr{P}$-minimal.

(ii) $(X, \mathscr{T})$ is first countable- and $\mathscr{P}$-closed.

(iii) $(X, \mathscr{T})$ is pseudocompact.

(iv) $(X, \mathscr{T})$ is countably compact.

Proof. It follows from Lemma 2.11 and an argument similar to the one given in the proof of Theorem 2.9 that (i) implies (ii).

If (ii) holds, then, according to Lemma 2.10 , every countable completely regular filter base on $(X, \mathscr{T})$ is fixed. As noted earlier, this latter property is a necessary and sufficient condition for pseudocompactness.

In $[10$, p. 232] a proof is given that (iii) and (iv) are equivalent on a weakly normal space.

It follows from Lemma 2.12 that (iv) implies (i).

COROLlary 2.14. A first countable paracompact space is first countable- and paracompact-minimal if and only if it is compact.

Proof. Every countably compact paracompact space is compact [1].

TheOREM 2.15. Let $(X, \mathscr{T})$ be a perfectly normal space. The following are equivalent:

(i) $(X, \mathscr{T})$ is minimal perfectly normal.

(ii) $(X, \mathscr{T})$ is perfectly normal-closed.

(iii) $(X, \mathscr{T})$ is first countable- and perfectly normal-minimal.

(iv) $(X, \mathscr{T})$ is first countable- and perfectly normal-closed.

(v) $(X, \mathscr{T})$ is pseudocompact.

(vi) $(X, \mathscr{T})$ is countably compact.

Proof. Since $(X, \mathscr{T})$ is perfectly normal, each of its points is a $G_{\delta} . \operatorname{In}[12$, p. 378] Glicksberg proved that every $G_{\delta}$ point in a pseudocompact completely regular space has a countable fundamental system of neighborhoods. Thus $(X, \mathscr{T})$ is first countable if either (v) or (vi) holds. In view of this, the proof of Theorem 2.13 can be used here to show that (iii), (iv), (v), and (vi) are equivalent. 
It follows from Lemma 2.11 that (i) implies (v).

(v) implies (i). Let $\mathscr{S}$ be a perfectly normal topology on $X$ such that $\mathscr{S} \subset \mathscr{T}$. Since the identity mapping of $(X, \mathscr{T})$ onto $(X, \mathscr{S})$ is continuous, $(X, \mathscr{S})$ is pseudocompact. Thus $(X, \mathscr{S})$ is first countable. Since (v) implies (iii), we must have $\mathscr{S}=\mathscr{T}$.

(v) implies (ii). Suppose that (v) holds. Then $(X, \mathscr{T})$ is first countable. Let $Y$ be a perfectly normal space in which $(X, \mathscr{T})$ is embedded, and let $q$ be a point in $\bar{X}$. Since $q$ is a $G_{\delta}$ and $Y$ is regular, we can construct a countable open filter base $\mathscr{D}$ on $Y$ such that $\bigcap \mathscr{D}=\bigcap\{\bar{C} \mid C \in \mathscr{D}\}=\{q\}$. Since $\mathscr{C}=\mathscr{D} \mid X$ is a countable open filter base on $(X, \mathscr{T})$ and $(X, \mathscr{T})$ is feebly compact, there exists $x \in X$ such that $x$ is an adherent point of $\mathscr{C}$. Therefore, $q=x \in X$.

An appropriate modification of the proof of Lemma 2.10 shows that (ii) implies (v).

Definition 2.16. A filter base on a topological space is called zero-dimensional if the sets belonging to it are open-and-closed sets.

Lemma 2.17. Let $X$ be a zero-dimensional space. The following are equivalent:

(i) $X$ is feebly compact.

(ii) Every countable zero-dimensional filter base on $X$ is fixed.

Proof. Obviously (i) implies (ii).

(ii) implies (i). Let $\mathscr{F}=\{F(n) \mid n \in N\}$ be a countable open filter base on $X$ which has no adherent point. For each $n$ let $G(n)=\bigcap\{F(i) \mid i \leqq n\}$, and let $\mathscr{G}=\{G(n) \mid n \in N\}$. Since $X$ is zero-dimensional and $\mathscr{G}$ is a filter base with empty adherence, we can choose a sequence of nonempty open-and-closed sets $O(n) \subset G(n)$ such that $O(i)$ $\cap O(j)=\varnothing$ whenever $i \neq j$. For each $n$ let $H(n)=\bigcup\{O(i) \mid i \geqq n\}$, and let

$$
\mathscr{H}=\{H(n) \mid n \in N\} .
$$

Because $\mathscr{G}$ has no adherent points, $\{O(j) \mid j \geqq n\}$ is locally finite for each $n \in N$. Thus each $\mathrm{Cl} H(n)=\bigcup\{\mathrm{Cl} O(j) \mid j \geqq n\}=\bigcup\{O(j) \mid j \geqq n\}=H(n)$, so $\mathscr{H}$ is a zerodimensional filter base which clearly has empty adherence.

Lemma 2.17 and appropriate modifications of Lemmas 2.10 and 2.11 can be used to obtain a proof of the next theorem.

THeorem 2.18. Let $X$ be a first countable zero-dimensional space. The following are equivalent:

(i) $X$ is first countable- and zero-dimensional-minimal.

(ii) $X$ is first countable- and zero-dimensional-closed.

(iii) $X$ is pseudocompact.

Definition 2.19. A topological space $X$ is called Urysohn if every pair of distinct points of $X$ have disjoint closed neighborhoods.

DEFinition 2.20. If $\mathscr{F}$ and $\mathscr{G}$ are filter bases on a set $X$, then $\mathscr{F}$ is said to be weaker than $\mathscr{G}$ provided that for every $F \in \mathscr{F}$ there is a set $G \in \mathscr{G}$ such that $F \supset G$. 
THEOREM 2.21. Let $X$ be a first countable Urysohn space. The following are equivalent:

(i) $X$ is first countable- and Urysohn-minimal.

(ii) Whenever $\mathscr{J}$ and $\mathscr{L}$ are arbitrary open filter bases on $X, \mathscr{K}$ is a countable closed filter base on $X, \mathscr{J}$ is weaker than $\mathscr{K}, \mathscr{K}$ is weaker than $\mathscr{L}$, and $\mathscr{J}$ has a unique adherent point, then $\mathscr{L}$ is convergent.

THEOREM 2.22. Let $X$ be a first countable Urysohn space. The following are equivalent:

(i) $X$ is first countable- and Urysohn-closed.

(ii) Whenever $\mathscr{J}$ and $\mathscr{L}$ are arbitrary open filter bases on $X, \mathscr{K}$ is a countable closed filter base on $X, \mathscr{J}$ is weaker than $\mathscr{K}$, and $\mathscr{K}$ is weaker then $\mathscr{L}$, then $\mathscr{J}$ has nonempty adherence.

THEOREM 2.23. A first countable- and Urysohn-minimal space $(X, \mathscr{T})$ is semiregular and first countable- and Urysohn-closed.

In [13] Herrlich proved theorems analogous to Theorems 2.21 and 2.22 for minimal Urysohn and Urysohn-closed spaces; he also proved that a minimal Urysohn space is Urysohn-closed. The proofs of Theorems 2.21 and 2.22 and the proof that first countable- and Urysohn-minimal implies first countable- and Urysohn-closed are similar to those in [13].

According to the Exercises in [8, pp. 167-168], $\left(X, \mathscr{T}_{s}\right)$ is a semiregular Urysohn space if $(X, \mathscr{T})$ is a Urysohn space. Since $\mathscr{T}_{s} \subset \mathscr{T}$, and $\left(X, \mathscr{T}_{s}\right)$ is first countable if $(X, \mathscr{T})$ is first countable, we must have $\mathscr{T}=\mathscr{T}_{s}$ if $(X, \mathscr{T})$ is first countable- and Urysohn-minimal, i.e., $(X, \mathscr{T})$ must be semiregular.

THEOREM 2.24. Let $X$ be a first countable regular space. The following are equivalent:

(i) $X$ is first countable- and Urysohn-minimal.

(ii) $X$ is first countable- and Urysohn-closed.

(iii) $X$ is first countable- and Hausdorff-minimal.

Proof. By Theorem 2.23, (i) implies (ii). It follows from (iii) of Theorem 2.6 and (ii) of Theorem 2.22 that (ii) implies (iii). Obviously (iii) implies (i).

Added in proof. In a forthcoming paper a space will be constructed which is first countable- and Urysohn-minimal, but not regular.

\section{Examples.}

3.1. Let $X$ be the set of all ordinal numbers less than the first uncountable ordinal, with the order topology. $X$ is first countable, locally compact, zerodimensional, completely normal [10, p. 146], and countably compact. Lemma 2.12 implies that $X$ is first countable- and $\mathscr{P}$-minimal for $\mathscr{P}=$ Hausdorff, regular, zerodimensional, etc. $X$ is not perfectly normal ([8] or Theorem 5.11). 
3.2. Let $\mathscr{M}$ be a maximal infinite family of infinite subsets of $N$ such that the intersection of any two is finite. Choose a new set of distinct points $D=\left\{d_{M} \mid M \in \mathscr{M}\right\}$, let $X=N \cup D$, and topologize $X$ as follows: the points of $N$ are isolated, and a neighborhood of a point $d_{M}$ is any set containing $d_{M}$ and all but finitely many elements of $M$. Then [11, p. 79] $X$ is a first countable completely regular space which is pseudocompact, but not countably compact. $X$ is also locally compact and zero-dimensional. Since a weakly normal pseudocompact space is countably compact [10], $X$ is not weakly normal. Theorems 2.6 and 2.9 imply that $X$ is first countable- and Hausdorff-minimal.

3.3. Choose two points $p, q$ not in the space $R$ of real numbers, let $X=R \cup\{p, q\}$, and let $\mathscr{T}$ be the topology on $X$ which has as a base

$$
\begin{aligned}
\{(a, b) \mid a, b \in R\} \cup\{\{p\} & \cup \cup\{(2 n, 2 n+1)|| n|\in N, k \leqq| n \mid\} \mid k \in N\} \\
& \cup\{\{q\} \cup \bigcup\{(2 n-1,2 n)|| n|\in N, k \leqq| n \mid\} \mid k \in N\} .
\end{aligned}
$$

In [16] it was proved that $(X, \mathscr{T})$ is minimal Hausdorff, but not compact. It is also true that $(X, \mathscr{T})$ is first countable, but neither Urysohn nor countably compact.

3.4. Let $X=[0,1]$, let $A$ be the set of rational numbers in $X$, and let $\mathscr{T}$ be the topology on $X$ which has as a base $\{\{A\} \cup\{X \cap(a, b) \mid a, b \in R\}\}^{\wedge}$. In [6] Berri noted that $(X, \mathscr{T})$ is absolutely closed (i.e., Hausdorff-closed), but not minimal Hausdorff. Thus $(X, \mathscr{T})$ is first countable- and Hausdorff-closed, but not first countable- and Hausdorff-minimal.

3.5. Let $X$ be as above, let $\mathscr{S}$ be the usual topology on $X$, and choose three dense subsets $X(1), X(2)$, and $X(3)$ of $(X, \mathscr{S})$ which are disjoint and whose union is $X$. Let $\mathscr{U}$ be the topology on $X$ generated by $\mathscr{S} \cup\{X(1), X(2)\}$. In [13] Herrlich noted that $(X, \mathscr{U})$ is Urysohn-closed, but neither minimal Urysohn nor absolutely closed. Since $(X, \mathscr{U})$ is second countable, it is also true that $(X, \mathscr{U})$ is first countableand Urysohn-closed, but neither feebly compact nor first countable- and Uyrsohnminimal. $(X, \mathscr{U})$ is clearly not regular, but it is semiregular: if $x \in X(i), i=1$ or 2 , and $a<x<b$, then $(a, b) \cap X(i)=\operatorname{Int}_{\mathscr{U}} \mathrm{Cl}_{\mathscr{U}}((a, b) \cap X(i))$; if $x \in X(3)$, then $(a, b) \cap X=\operatorname{Int}_{\mathscr{U}} \mathrm{Cl}_{\mathscr{U}}((a, b) \cap X)$. Thus the converse of Theorem 2.23 is false.

4. Products. Let $M$ be a (nonempty) subset of $N$.

THEOREM 4.1. Let $\{X(n) \mid n \in M\}$ be a collection of topological spaces and $X=\prod X(n)$. Then $X$ is first countable-and Hausdorff-closed if and only if each $X(n)$ is first countable- and Hausdorff-closed.

Proof. Since $X$ is first countable if and only if each $X(n)$ is first countable, it follows from Theorems 4.2 and 4.4 in [15] that $X$ is feebly compact if and only if each $X(n)$ is feebly compact.

LEMMA 4.2. A countably compact completely regular space $X$ is weakly normal.

Proof. Let $F$ and $C$ be disjoint closed subsets of $X$ such that $C$ is countable. Since $X$ is countably compact, $C$ must be compact. In any completely regular 
space, two disjoint closed sets, one of which is compact, have disjoint neighborhoods [11, p. 42].

THEOREM 4.3. Let $\{X(n) \mid n \in M\}$ be a collection of topological spaces, let $X=\prod X(n)$, and let $\mathscr{P}$ be any one of Hausdorff, regular, completely regular, zerodimensional, and weakly normal. Then $X$ is first countable-and $\mathscr{P}$-minimal if and only if each $X(n)$ is first countable- and $\mathscr{P}$-minimal.

Proof. For $\mathscr{P}=$ Hausdorff, regular, completely regular, and zero-dimensional, the theorem is a consequence of Theorems 2.4, 2.6, 2.9, 2.18, and 4.1 and the fact that if $\mathscr{S}=$ semiregular [19, p. 45], regular, completely regular, or zero-dimensional, then $X$ is an $\mathscr{S}$-space if and only if each $X(n)$ is an $\mathscr{S}$-space.

If $X$ is first countable- and weakly normal-minimal, then $X$ is first countable, completely regular, and countably compact (Theorem 2.13 ), so each $X(n)$ must be first countable, completely regular, and countably compact. It follows from Lemmas 2.12 and 4.2 that each $X(n)$ is first countable- and weakly normal-minimal.

If each $X(n)$ is first countable- and weakly normal-minimal, then each $X(n)$ is first countable and countably compact, so $X$ is first countable and countably compact. Since $X$ is also completely regular, Lemmas 2.12 and 4.2 imply that $X$ is first countable- and weakly normal-minimal.

THEOREM 4.4. Let $\mathscr{P}$ denote either of normal and completely normal, and let $\{X(n) \mid n \in M\}$ be a collection of spaces.

(i) If $X=\prod X(n)$ is a $\mathscr{P}$-space and each $X(n)$ is first countable- and $\mathscr{P}$-minimal, then so is $X$.

(ii) If $X$ is first countable-and $\mathscr{P}$-minimal, then so is each $X(n)$.

THEOREM 4.5. Let $\{X(n) \mid n \in M\}$ be a collection of spaces. If $X=\prod X(n)$ is minimal perfectly normal, then so is each $X(n)$. If $X$ is perfectly normal and each $X(n)$ is minimal perfectly normal, then $X$ is minimal perfectly normal.

As far as the author knows, the following question is open: What are necessary and sufficient conditions that the product of a countable collection of spaces be first countable- and Urysohn-minimal (Urysohn-closed)? The next few results give some partial answers.

THEOREM 4.6. If $\{X(n) \mid n \in M\}$ is a collection of spaces such that $\Pi X(n)$ is first countable- and Urysohn-minimal, then each $X(n)$ is a first countable- and Urysohn-minimal space.

This is an immediate consequence of the fact that a collection $\{X(a) \mid a \in A\}$ of spaces has a Urysohn product if and only if each $X(a)$ is a Urysohn space.

THEOREM 4.7. If $\{X(n) \mid n \in M\}$ is a collection of spaces such that $\Pi X(n)$ is first countable- and Urysohn-closed, then each $X(n)$ is a first countable- and Urysohnclosed space. 
More generally, it follows easily from (ii) of Theorem 2.22 that if $Y$ and $Z$ are first countable Urysohn spaces, $f \in C(Y, Z)$, and $Y$ is first countable- and Urysohnclosed, then so is $f(Y)$.

THEOREM 4.8. If $X$ and $Y$ are first countable- and Urysohn-closed spaces and $X$ is Hausdorff-closed, then $X \times Y$ is a first countable- and Urysohn-closed space.

Proof. We first note that for every open subset $A$ of $X \times Y, \operatorname{pr}_{2}(\bar{A})$ is closed (see [15, Theorem 2.17]).

Let $\mathscr{J}$ and $\mathscr{L}$ be open filter bases on $X \times Y$ such that $\mathscr{J}$ is weaker than the countable filter base $\mathscr{K}=\{\bar{L} \mid L \in \mathscr{L}\}$. Then $\operatorname{pr}_{2}(\mathscr{J}), \operatorname{pr}_{2}(\mathscr{K})$, and $\operatorname{pr}_{2}(\mathscr{L})$ satisfy the hypothesis of (ii) of Theorem 2.22 , so $\operatorname{pr}_{2}(\mathscr{J})$ has an adherent point $y$. Let $\mathscr{C}$ be a countable fundamental system of open neighborhoods of $y$, and let

$$
\mathscr{D}=\{J \cap(X \times C) \mid J \in \mathscr{J} \text { and } C \in \mathscr{C}\} \text {. }
$$

Then $\operatorname{pr}_{1}(\mathscr{D})$ is clearly an open filter base and hence has an adherent point $x$. The point $(x, y)$ is an adherent point of $\mathscr{J}$.

THEOREM 4.9. If $X$ and $Y$ are first countable- and Urysohn-minimal spaces and $X$ is compact, then $X \times Y$ is a first countable- and Urysohn-minimal space.

Proof. We prove that (ii) of Theorem 2.21 holds. Let $\mathscr{J}$ and $\mathscr{L}$ be open filter bases on $X \times Y$ such that $\mathscr{L}$ is countable, $\mathscr{J}$ has a unique adherent point, say $(x, y)$, and $\mathscr{J}$ is weaker than $\mathscr{K}=\{\bar{L} \mid L \in \mathscr{L}\}$. We wish to show that $\mathscr{L}$ is convergent.

Since $(x, y)$ is an adherent point of $\mathscr{J}$, and $\mathrm{pr}_{2}$ is continuous, $y$ is an adherent point of $\operatorname{pr}_{2}(\mathscr{J})$. In fact, $y$ is the only adherent point of $\operatorname{pr}_{2}(\mathscr{J})$, for suppose $z \neq y$ and $z$ is an adherent point of $\operatorname{pr}_{2}(\mathscr{J})$. Then by an argument similar to one given in the proof above, we can show that there is a point $t$ in $X$ such that $(t, z)$ is an adherent point of $\mathscr{J}$, whereas $\mathscr{J}$ has no adherent point other than $(x, y)$. Thus $\operatorname{pr}_{2}(\mathscr{J})$ has a unique adherent point. Since $X$ is countably compact and $Y$ is first countable, $\mathrm{pr}_{2}$ is a closed mapping. Thus $\mathrm{pr}_{2}(\mathscr{J}), \mathrm{pr}_{2}(\mathscr{K})$, and $\operatorname{pr}_{2}(\mathscr{L})$ satisfy the hypothesis of (ii) of Theorem 2.21. Since $Y$ is first countable- and Urysohn-minimal, $\operatorname{pr}_{2}(\mathscr{L})$ is convergent.

Consider the countable filter base $\operatorname{pr}_{1}(\mathscr{L})$. Since $X$ is countably compact, $\operatorname{pr}_{1}(\mathscr{L})$ has an adherent point. Furthermore, it can be shown that $\operatorname{pr}_{1}(\mathscr{L})$ has at most one adherent point, for suppose that $x_{1}, x_{2}$ are adherent points $\operatorname{of} \mathrm{pr}_{1}(\mathscr{L})$. Set $\mathscr{L}(i)=\left\{L \cap(V(i) \times Y) \mid L \in \mathscr{L}\right.$ and $V(i)$ is a neighborhood of $\left.x_{i}\right\}, i=1,2$. Then $\operatorname{pr}_{2}(\mathscr{L}(1))$ and $\operatorname{pr}_{2}(\mathscr{L}(2))$ are filter bases on $Y$ stronger than $\operatorname{pr}_{2}(\mathscr{L})$, so each $\operatorname{pr}_{2}(\mathscr{L}(i))$ is convergent. Since the adherence of $\operatorname{pr}_{2}(\mathscr{L}(i))$ is contained in $\{y\}, \operatorname{pr}_{2}(\mathscr{L}(i))$ converges to $y$. Therefore, $\left(x_{i}, y\right)$ is an adherent point of $\mathscr{L}$ and, consequently, an adherent point of $\mathscr{J}$, so $x_{i}=x, i=1,2$. Thus $x$ is the unique adherent point of $\operatorname{pr}_{1}(\mathscr{L})$. It follows from this that $\operatorname{pr}_{1}(\mathscr{L})$ is convergent, for on a countably compact space a countable filter base with a unique adherent point is convergent.

Since $\operatorname{pr}_{1}(\mathscr{L})$ and $\operatorname{pr}_{2}(\mathscr{L})$ are convergent, $\mathscr{L}$ is convergent. 
5. Subspaces. In [1] it is proved that a space $X$ is feebly compact if and only if the closure of every open subset is feebly compact.

THEOREM 5.1. A space $X$ is first countable- and Hausdorff-closed if and only if the closure of every open subset of $X$ is first countable- and Hausdorff-closed.

The following theorems are immediate consequences of this result and the characterization theorems obtained in $\S 2$.

THEOREM 5.2. Let $X$ be a first countable-and Hausdorff-minimal space, and let $A$ be an open subset of $X$. Then $\bar{A}$ is first countable-and Hausdorff-minimal if and only if it is semiregular.

REMARK 5.3. If in Example 3.3 one takes $A=\bigcup\{(2 n, 2 n+1) \mid n \in N\}$, then $A$ is open, but $\bar{A}$ is not semiregular.

TheOREM 5.4. Let $\mathscr{P}$ denote any one of regular, completely regular, and zerodimensional, and let $X$ be a first countable- and $\mathscr{P}$-minimal space. If $A$ is an open subset of $X$, then $\bar{A}$ is first countable-and $\mathscr{P}$-minimal.

EXAMPLE 5.5. The closure of an open subset of a first countable- and Urysohnclosed space need not be first countable- and Urysohn-closed. In the space $(X, \mathscr{U})$ in Example 3.5 let $A=X(1)$. Then $A$ is open and $\bar{A}=X(1) \cup X(3)$. Fix $x \in X(2)$ and let $\mathscr{R}=\{\bar{A} \cap(x-1 / n, x+1 / n) \mid n \in N\}$. Then $\mathscr{R}$ is a free, countable, regular filter base on $\bar{A}$, so it follows from Theorem 2.22 that $\bar{A}$ cannot be first countableand Urysohn-closed.

As the following theorem shows, it is not, in general, true that every closed subset of a first countable- and $\mathscr{P}$-minimal space is first countable- and $\mathscr{P}$-minimal.

THEOREM 5.6. A Hausdorff space $X$ is countably compact if and only if every countable closed subset of $X$ is feebly compact.

Proof. If $X$ is countably compact, then every closed subset of $X$ is countably compact and hence feebly .compact. Conversely, if $C$ is a countably infinite subset of $X$ which has no limit point, then $C$ is a closed subset of $X$ which is an infinite discrete space.

In [2] Banaschewski proved that a Hausdorff space can be embedded densely in a minimal Hausdorff space if and only if it is semiregular. In [18] Strecker and Wattel proved that every Hausdorff space can be embedded in a minimal Hausdorff space. The next three theorems give similar results for first countable- and Hausdorff-minimal spaces.

THEOREM 5.7. Let $X$ be a first countable Hausdorff space, let $\mathscr{N}=\{\mathscr{F} \mid \mathscr{F}$ is a countable open filter base on $X$ which has no adherent points $\}$, and let $\mathscr{M}$ be a subset of $\mathscr{N}$ which is maximal with respect to having the property that whenever $\mathscr{F}, \mathscr{G} \in \mathscr{M}$, $\mathscr{F} \neq \mathscr{G}$, then there exist sets $F \in \mathscr{F}$ and $G \in \mathscr{G}$ such that $F \cap G=\varnothing$. Let $D=\{p(\mathscr{F}) \mid \mathscr{F} \in \mathscr{M}\}$ be a new set of distinct points, and let $Y=X \cup D$, topologized 
as follows: a set $O \subset Y$ is open if and only if (a) $O \cap X$ is open in $X$, and (b) if $p(\mathscr{F}) \in O$, then $O \cap X$ contains a set belonging to $\mathscr{F}$. Then $Y$ is a first countable- and Hausdorffclosed space in which $X$ is embedded as a dense subspace.

The proof is straightforward.

Remark 5.8. If $X$ is a first countable- and Hausdorff-closed space which is not absolutely closed (e.g., 3.1), then $X$ cannot be embedded densely in a first countable absolutely closed space.

THEOREM 5.9. Let $X$ be a first countable Hausdorff space, and let $(Y, \mathscr{T})$ be the first countable- and Hausdorff-closed space given by Theorem 5.7. The following are equivalent.

(i) $X$ is semiregular.

(ii) $\left(Y, \mathscr{T}_{s}\right)$ is a first countable- and Hausdorff-minimal space in which $X$ is embedded as a dense subspace.

Proof. $\left(Y, \mathscr{T}_{s}\right)$ is first countable- and Hausdorff-minimal by Theorem 2.5. Banaschewski's computations in [4, pp. 146-147] show that if $X$ is any space embedded densely in a space $(Y, \mathscr{T})$, then semiregularity is a sufficient condition that $X$ be embedded densely in $\left(Y, \mathscr{T}_{s}\right)$.

Lemma 1 of [2] implies that a dense subspace of a semiregular space is semiregular. Thus if $X$ is embedded densely in $\left(Y, \mathscr{T}_{s}\right)$, a semiregular space, then $X$ must be semiregular.

THEOREM 5.10. A first countable Hausdorff space can be embedded in a first countable- and Hausdorff-minimal space.

Proof. In [18, p. 235] Strecker and Wattel proved that every Hausdorff space can be embedded in a semiregular Hausdorff space. Their method of proof shows that every first countable Hausdorff space can be embedded in a first countable, semiregular, Hausdorff space $Z$. By Theorem $5.9, Z$ can be embedded in a first countable- and Hausdorff-minimal space.

The author does not know for what other properties $\mathscr{P}$ it is true that every first countable $\mathscr{P}$-space can be embedded in a first countable- and $\mathscr{P}$-minimal space. Example 3.5 does show that there exists a semiregular, first countable, Urysohn space which cannot be embedded densely in a first countable- and Urysohnminimal space (it is first countable- and Urysohn-closed, but not first countableand Urysohn-minimal).

The next theorem shows that not every perfectly normal space can be embedded in a minimal perfectly normal space.

THEOREM 5.11. Let $X$ be a countably compact perfectly normal space and suppose that $D$ is a discrete subset of $X$. Then $D$ is countable.

Proof. $D$ is a dense subset of $\bar{D}$, so every isolated point of $D$ is isolated in $\bar{D}$. Thus $D$ is open in $\bar{D}$. Since $\bar{D}$ is perfectly normal, there exist closed sets $F(n)$, 
$n \in N$, such that $D=\bigcup\{F(n)\}$. Because $X$ is countably compact, each $F(n)$ is a countably compact discrete space. Hence each $F(n)$ is finite and $D$ is countable.

6. Category. An immediate consequence of the proof of Theorem 6 in [5] is the following:

THEOREM 6.1. Let $\mathscr{P}$ denote any one of regular, completely regular, zerodimensional, weakly normal, normal, completely normal, and perfectly normüi, and let $X$ be a first countable- and $\mathscr{P}$-mimimal space. Then $X$ is second category.

An example of Herrlich (Math. Z. 91 (1966), 185) shows that a first countableminimal Hausdorff space need not be second category. The author does not know if every first countable- and Urysohn-minimal space is second category.

\section{REFERENCES}

1. R. W. Bagley, E. H. Connell and J. D. McKnight, Jr., On properties characterizing pseudocompact spaces, Proc. Amer. Math. Soc. 9 (1958), 500-506.

2. B. Banaschewski, Über Hausdorffsch-minimale Erweiterung von Räumen, Arch. Math. 12 (1961), 355-365.

3. - Über nulldimensionale Räume, Math. Nachr. 13 (1955), 129-140.

4. - Über zwei Extremaleigenschaften topologischer Räume, Math. Nachr. 13 (1955), 141-150.

5. M. P. Berri, Categories of certain minimal topological spaces, J. Austral. Math. Soc. 4 (1964), 78-82.

6. - Minimal topological spaces, Trans. Amer. Math. Soc. 108 (1963), 97-105.

7. M. P. Berri and R. H. Sorgenfrey, Minimal regular spaces, Proc. Amer. Math. Soc. 14 (1963), 454-458.

8. N. Bourbaki, Topologie générale, 3rd ed., Actualités Sci. Indust. No. 1142, Hermann, Paris, 1961.

9. H. H. Corson and E. A. Michael, Metrizability of certain countable unions, Illinois J. Math. 8 (1964), 351-360.

10. J. Dugundji, Topology, Allyn and Bacon, Boston, Mass., 1966.

11. L. Gillman and M. Jerison, Rings of continuous functions, Van Nostrand, New York, 1960.

12. I. Glicksberg, Stone-Čech compactifications of products, Trans. Amer. Math. Soc. 90 (1959), 369-382.

13. H. Herrlich, $T_{v}$-Abgeschlossenheit und $T_{v}$-Minimalität, Math. Z. 88 (1965), 285-294.

14. C. T. Scarborough and R. M. Stephenson, Jr., Minimal topologies; Colloq. Math. 19 (1968), 215-219.

15. C. T. Scarborough and A. H. Stone, Products of nearly compact spaces, Trans. Amer. Math. Soc. 124 (1966), 131-147.

16. N. Smythe and C. A. Wilkins, Minimal Hausdorff and maximal compact spaces, J. Austral. Math. Soc. 3 (1963), 167-171.

17. R. M.Stephenson, Jr., Pseudocompact spaces, Trans. Amer. Math. Soc. 134 (1968), 437-448.

18. G. E. Strecker and E. Wattel, On semi-regular and minimal Hausdorff embeddings, Proc.

Roy. Netherlands Acad. Sci. A70 (1967), 234-237; Indag. Math. 29 (1967), 234-237.

19. G. E. Strecker, Co-topologies and generalized compactness conditions, Dissertation, Tulane University, New Orleans, La., 1966.

University of North Carolina, Chapel Hill, North Carolina 